\title{
reviscafuences
}

ISSN: 1575-7072 | e-ISSN: 2172-7775

Páginas: 142-152

Recibido: 2019-10-14

Revisado: 2020-02-17

Aceptado: 2020-04-01

Preprint: 2020-07-15

Publicación Final: 2020-09-15 www.revistascientificas.us.es/index.php/fuentes/index

DOI: https://doi.org/10.12795/revistafuentes.2020.v22.i2.05

\section{Intención de abandonar la carrera: Influencia de variables personales y familiares}

\section{Dropout Intention at University: Influence of Personal and Family Variables}

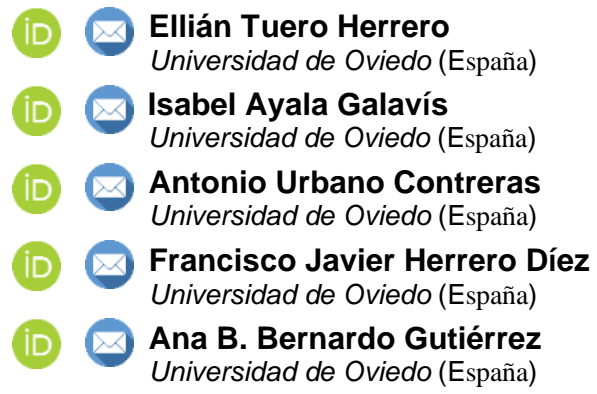

\section{Resumen}

Múltiples investigaciones en el campo del abandono de los estudios resaltan el impacto que pueden tener las variables personales del estudiante. Sin embargo, pocas se han detenido a investigar la influencia que puede tener la familia en el progreso académico del alumnado universitario, analizando el papel que esta pueda desempeñar en la intención de que un joven tome la decisión de permanecer o abandonar sus estudios. Con el objeto de profundizar en ello, y en la relación existente entre algunas variables de la esfera familiar y personal del estudiante, se lleva a cabo un estudio con 692 alumnos de una universidad del norte de España. Para la recogida de datos se administró un cuestionario con las dimensiones que recogían las variables de interés. Los resultados ponen de manifiesto la relación entre las variables de ambas esferas y muestran que el contexto familiar tiene influencia durante la etapa educativa universitaria. Los estudiantes que sienten ansiedad ante la idea de comunicar a sus familias la intención de cambiar o dejar la carrera son los que tienen más posibilidades de que, finalmente, se planteen abandonar. Por contra, aquellos que manifiestan no haber elegido la titulación por presión familiar y que perciben una implicación positiva en sus estudios son los que mayor intención tienen de permanecer. Estos hallazgos demuestran la urgente necesidad de llevar a cabo intervenciones educativas preventivas a nivel familiar.

\begin{abstract}
A wide range of research on dropping out of studies has remarked the influence of the student's personal variables. Nonetheless, little studies are dedicated to investigate the family influence on the academic progress during the university stage, especially the role it could play in the students' decision on staying or abandoning their career. In order to deeply study this issue and the existing relationship between certain variables within the personal and family circle of the students, a questionnaire has been conducted with a sample of 692 students from a Spanish northern university. The data collection was carried out with a questionnaire made up with the dimensions covering the variables of interest. Results have proven the relationship between variables within both circles, and also that family context has influence during the university stage. Students who feel anxious about communicating to their families the intention of changing their career or leaving it permanently are the most likely to eventually consider dropping out their studies. In contrast, those students who have not chosen their career due to family pressure and perceive family support are those who are more likely to continue their studies selection. These findings highlight the compelling need to implement preventive educational interventions at a family level.
\end{abstract}

\section{Palabras clave / Keywords}

Educación superior, abandono universitario, permanencia, influencia familiar. Higher education, university dropout, student's persistence, family influence. 


\section{Introducción}

El creciente número de casos de estudiantes que abandonan la universidad sin haber culminado sus estudios es un hecho que cada vez suscita mayor preocupación por parte de los estados y las instituciones educativas, no solo por la importante pérdida de recursos que implica, sino también por las limitaciones que supone para el desarrollo social, económico y cultural de la sociedad (González \& Uribe, 2018). En este escenario, los primeros afectados son los estudiantes y sus familias quienes, en muchos casos, deben lidiar tanto con los costes económicos, como con los sentimientos de frustración y decepción derivados de tal decisión (De Ortuzar \& Di Meglio, 2016; Nervi, Rodríguez, \& Osada, 2015). Como resultado de ello, la reducción del abandono universitario se torna como uno de los objetivos prioritarios dentro de las agendas gubernamentales. De hecho, y como meta a alcanzar, se ha proyectado en la Estrategia Europa 2020 reducir las tasas actuales a un 10\% (Comisión Europea, 2012). Para ello, tal y como reflejan distintos investigadores (Álvarez-Pérez \& López-Aguilar, 2017; Iñíguez, Elboj, \& Valero, 2016), es indispensable seguir ahondando en su conocimiento, con vistas a lograr dicho objetivo y desarrollar las medidas preventivas oportunas.

Desde la notable aparición de este fenómeno, con Tinto (1975), algunos autores han ido elaborando múltiples modelos explicativos que posibilitan comprender la naturaleza y complejidad que lo caracterizan. Si bien, todos ellos llegan al acuerdo de que se trata de un fenómeno multicausal, cada uno se centra en otorgar más énfasis a algunas variables frente a otras (individuales, institucionales o del medio familiar), resultando así diversos enfoques, a saber: psicológico, económico, sociológico, organizacional e interaccionista. Sin embargo, es este último el que ha abordado su estudio desde una perspectiva más integradora, al analizar una gran diversidad de variables, permitiendo, además, determinar la importancia que tiene cada una de ellas en la decisión de un alumno de abandonar sus estudios superiores (García \& Adrogué, 2015).

Así, dentro de las variables personales del alumnado, se ha demostrado que el rendimiento académico previo al ingreso a la universidad (Rodrigo, Molina, García-Ros, \& Pérez-González, 2012) y durante el primer año de carrera es una de las que mayor influencia tiene en el planteamiento y consolidación del abandono (Eckert \& Suénaga, 2015). Los resultados obtenidos por Bernardo et al. (2015) indican que los alumnos que abandonan, a diferencia de los que permanecen en sus estudios, son aquellos que presentan menores puntuaciones medias tanto en la nota de acceso a la titulación como en el número de créditos aprobados en la carrera.

Las variables personales de carácter afectivo-motivacional también mantienen una estrecha relación con el abandono. Por ejemplo, la elección de la carrera por vocación es una variable importante en la decisión de permanecer en la universidad como así lo demuestra el estudio predictivo realizado por Esteban, Bernardo, Tuero, Cervero y Casanova (2017). Esta investigación, muestra que los estudiantes que afirman haber elegido la titulación que están cursando principalmente por vocación, tienen el doble de probabilidades de permanecer en la misma (odds ratio $=2.099$ ) en comparación con aquellos que han dado más peso a otros motivos. Asimismo, otros investigadores como Girelli et al. (2018) han encontrado que los alumnos que deciden estudiar en la universidad por motivos intrínsecos (por decisión y voluntad propia, por agrado hacia la titulación elegida, o por interés y satisfacción personal derivados) en lugar de fundamentar su decisión principalmente en las opiniones, expectativas o recomendaciones de otras personas (padres, familiares cercanos, orientadores, etc.) son menos propensos a dejar sus estudios.

En este mismo grupo de variables, como así lo comprueban Bethencourt, Cabrera, Hernández, Álvarez y González (2008), la satisfacción con la titulación cursada $(\eta 2=.358)$ y la motivación hacia la misma $(\eta 2=.220)$ son también relevantes para explicar las diferencias existentes entre los estudiantes que culminan sus estudios frente a aquellos que los abandonan. Los resultados obtenidos por estos autores reflejan que estas dos variables psicológicas, junto con otras tres ("persistencia para acabar los estudios a pesar de los obstáculos", "capacidad para demorar las recompensas" y "ajuste entre capacidades y exigencias de la titulación"), permiten explicar el $58 \%$ de las diferencias entre ambos grupos. Resultados que también están en línea con los obtenidos en investigaciones previas realizadas por estos mismos autores (González, Álvarez, Cabrera, \& Bethencourt, 2007).

El grado de integración con la institución es otro aspecto ampliamente estudiado dada su influencia en la permanencia del alumnado en la universidad. El estudio realizado por Motl, Multon y Zhao (2018), revela que algunas variables relacionadas con la integración, tanto en su dimensión académica como social, juegan un papel importante en la predicción de la persistencia universitaria. Concretamente, el tener una buena preparación académica previa, obtener un buen rendimiento en la carrera, tener sentido de pertenencia a la institución, percibir que se recibe un trato justo y honesto de parte de los compañeros, profesores y demás miembros de la institución, disfrutar de la atmósfera del campus y sus actividades, entre otras; permiten explicar en un $72 \%$ la persistencia del alumnado. En relación con este grupo de variables sociales, otra que 
se ha considerado relevante en la predicción de la permanencia y el abandono universitario es la asistencia a clases. El estudio realizado por Cabrera, Bethencourt, González y Álvarez (2006) refleja que el $96.3 \%$ de los alumnos que habían finalizado sus estudios manifestaron haber asistido a clase con regularidad. Resultados similares obtuvieron Bernardo et al. (2015) quienes, además, ponen de relieve la importancia que dicha variable tiene en la adaptación académica y social, ya que no solo favorece el proceso de aprendizaje, sino que también contribuye positivamente a la integración del estudiante con sus compañeros y profesores. Con respecto a las variables familiares en la educación superior, se ha demostrado que cuanto mayor es el nivel socio-económico y educativo de los padres menor es la tendencia de los jóvenes a abandonar sus estudios universitarios (García \& Adrogué, 2015; Ghignoni, 2017). No obstante, estas variables, en comparación con aquellas relativas al alumno (número de créditos aprobados en primer curso, elección de carrera por vocación, adaptación académica universitaria, tiempo dedicado al estudio) presentan un tamaño del efecto pequeño $(n 2 p=.013)$ como así han mostrado algunos estudios (Esteban, Bernardo, Tuero, Cerezo \& Núñez, 2016).

Ahora bien, continuando con este grupo de estudios que relacionan las variables familiares y el abandono de los estudios universitarios, cabe decir que no hay un elevado número de investigaciones en el contexto universitario que, más allá de las características socio-demográficas, hayan puesto su foco de interés en el estudio de otros factores. En este sentido, poco se ha analizado sobre la influencia que, por ejemplo, tienen: el apoyo, las expectativas y las aspiraciones de los padres en torno a las decisiones académico-profesionales de sus hijos, o bien, los efectos que el soporte emocional de éstos puede tener para que los jóvenes hagan frente a los retos y exigencias que implica la vida universitaria.

Dentro de estas investigaciones, la realizada por Stubbs y Maynard (2017) pone de manifiesto la importancia que tiene la familia en el progreso académico de los jóvenes en la etapa de transición a la universidad. En este estudio, llevado a cabo con una muestra de alumnos con edades comprendidas entre los 17 y 19 años, estos autores observaron que aquellos que pertenecían a familias más cohesionadas mostraban un mayor grado de engagement en sus estudios frente a aquellos otros cuyas familias presentaban niveles más bajos de cohesión. Además, los resultados obtenidos en dicho estudio indican que si bien el funcionamiento familiar no explica un elevado porcentaje de varianza (13.5\%), el nivel de cohesión sí que tiene un peso significativo a la hora de predecir el grado de implicación que manifiestan los jóvenes en su aprendizaje y actividades académicas $(\beta=.361)$. En esta misma línea, también se ha demostrado que el apoyo familiar juega un rol importante en la persistencia académica. En efecto, Ojeda, Navarro y Morales (2011), reflejan que mientras más perciben los jóvenes que sus padres les apoyan, alientan y estimulan a obtener una formación universitaria mayor intención tienen estos de permanecer en sus estudios. Asimismo, la investigación realizada por Strom y Savage (2014) demuestra que este tipo de apoyo familiar favorece el nivel de compromiso académico que desarrollará el estudiante durante el primer año de carrera, con un tamaño del efecto grande $(\beta=.72)$.

A tenor de lo expuesto, y dada la influencia que presenta la familia en el progreso académico de los jóvenes, el presente estudio tiene como objetivo analizar qué papel juega ésta en el planteamiento del abandono durante la educación superior. Para dar respuesta a este objetivo serán medidas diversas variables de la esfera personal (rendimiento académico previo al ingreso, motivos de elección de la titulación y nivel de adaptación a la vida universitaria) y familiar del alumnado (datos socio-demográficos, percepción de apoyo de la familia y expectativas familiares), así como la relación existente entre ellas y la intención de abandonar la titulación.

\section{Metodología}

\subsection{Diseño}

En la presente investigación se ha llevado a cabo un estudio bajo un diseño de investigación ex-post-facto, descriptivo y predictivo.

\subsection{Participantes}

Han participado 692 alumnos universitarios de primer año de carrera, 481 mujeres (69.5\%) y 211 hombres $(30.5 \%)$, con edades comprendidas entre 17 y 55 años $(M=19.81$, DT $=4.27)$ y pertenecientes a varias titulaciones (Enfermería, Psicología, Economía, Derecho, Administración y Dirección de Empresa, Química y Magisterio Primaria) de una universidad pública del norte de España. 


\subsection{Instrumento}

La recogida de información se realizó mediante un cuestionario creado específicamente por los autores. En este sentido, la construcción del mismo fue inicialmente realizada a través de estudios previos, los cuales permitieron ir perfeccionando el instrumento al recoger una amplia información sobre la multiplicidad de variables influyentes en este fenómeno; concretamente: variables sociodemográficas; rendimiento académico previo; motivos de elección de la titulación; disponibilidad de recursos para costear los estudios; grado de adaptación académica; grado de integración social en la institución; ansiedad del estudiante ante responsabilidades y decisiones académicas; intención de abandono de los estudios; influencia familiar en la elección de la titulación, incidencia de la familia en la toma de decisiones, sus expectativas y el apoyo familiar. El instrumento presenta un formato de respuesta tipo Likert de 1 a 5 puntos (1=totalmente en desacuerdo, $2=$ en desacuerdo; $3=$ ni en desacuerdo ni de acuerdo, $4=$ de acuerdo y $5=$ totalmente de acuerdo) presentado un índice de fiabilidad de .78; valor considerado aceptable según Cronbach y Shavelson (2004).

\subsection{Procedimiento y análisis de datos}

Se optó por un muestreo no probabilístico, de tipo incidental, accediendo a la misma por conveniencia; contactando con todo el profesorado que, finalmente, se mostró predispuesto a colaborar tras explicarse en detalle el objetivo de investigación. Los cuestionarios fueron aplicados de dos formas. En la primera, los estudiantes rellenaron el instrumento en papel durante 25 minutos en horario lectivo. En todo momento se encontraban en el aula el profesor-colaborador que impartía la docencia y un miembro del equipo de investigación. En cuanto a la segunda, se rellenó en formato virtual proporcionando un link al alumnado. La recogida de información tuvo lugar a finales del primer semestre del curso 2018/19, momento clave según la evidencia científica para decidir quedarse realizando el grado o plantearse abandonarlo (Silva, 2011).

Para el análisis de los datos se ha utilizado el software estadístico SPSS versión 23. Los análisis efectuados incluyen un bloque de resultados descriptivos y contrastes de medias en función de las variables de clasificación de la muestra. Las diferencias significativas se analizaron con el estadístico de contraste t de Student y el tamaño del efecto se indica con la d de Cohen. Además, con el fin de estudiar la relación entre variables, se ha calculado la correlación de Pearson, asegurando que sus valores fueran menores a .70 y descartando así la multicolinealidad. Adicionalmente, se desarrollaron dos modelos predictivos calculados mediante regresión lineal a través del método "paso a paso" con el fin de determinar el peso explicativo de unas variables sobre otras.

\section{Resultados}

Analizando los resultados globales (ver Tabla 1), en lo que respecta a la influencia familiar en la etapa previa al ingreso, se observa que hay una tendencia en el alumnado universitario a elegir la carrera por voluntad propia y no por algún tipo de presión o tradición familiar; descartando que la familia se hubiera sentido desilusionada en caso de haber elegido otra. Por otro lado, y ya en la etapa universitaria propiamente, una vez que los alumnos están cursando la carrera elegida, se observa una tendencia a considerar que la familia les anima y se muestra optimista respecto a su futuro académico, se siente orgullosa de lo que hacen y de sus planes futuros, y les apoya y confía en sus decisiones. 
Tabla 1

Resultados descriptivos. Media, desviación típica, mediana y rango intercuartílico

\begin{tabular}{|c|c|c|c|c|}
\hline & $\mathrm{M}$ & DT & Mdn & IQR \\
\hline 1.Mi familia ha ejercido algún tipo de presión en la elección de esta carrera & 1.5 & .94 & 1 & 1 \\
\hline 2. He elegido mi carrera por tradición familiar & 1.3 & .78 & 1 & 0 \\
\hline 3.La elección de carrera ha sido exclusivamente mía & 4.5 & .84 & 5 & 1 \\
\hline 4.Es un gran esfuerzo para mi familia costear mis estudios & 3.2 & 1.14 & 3 & 2 \\
\hline 5.Me siento satisfecho/a con la elección de mi titulación & 4.3 & .74 & 4 & 1 \\
\hline $\begin{array}{l}\text { 6. Mi asistencia a clase es elevada, acudo a la mayoría de las clases en la } \\
\text { Universidad }\end{array}$ & 4.4 & .86 & 5 & 1 \\
\hline 7.Me produce ansiedad pensar en ir a la facultad & 2.0 & 1.02 & 2 & 2 \\
\hline 8.Mi nivel de adaptación en el ámbito académico es satisfactorio & 4.1 & .71 & 4 & 1 \\
\hline 9.Mi nivel de adaptación en el ámbito social es satisfactorio & 4.2 & .76 & 4 & 1 \\
\hline 10.Pienso, habitualmente, en abandonar mis estudios y la titulación actual & 1.7 & .97 & 1 & 1 \\
\hline 11. Mi familia me anima y se muestra optimista respecto a mi futuro académico & 4.4 & .78 & 5 & 1 \\
\hline $\begin{array}{l}\text { 12.Mi familia se siente orgullosa de lo que estoy haciendo y de mis planes } \\
\text { futuros }\end{array}$ & 4.4 & .79 & 5 & 1 \\
\hline 13.Mi familia me apoya y confía en mis decisiones & 4.4 & .78 & 5 & 1 \\
\hline 14.Mi éxito y/o fracaso académico también es el de mi familia & 3.0 & 1.23 & 3 & 2 \\
\hline 15.Mi familia tiene expectativas muy elevadas sobre mi futuro académico & 3.7 & .94 & 4 & 1 \\
\hline 16.Si hubiese elegido otra carrera mi familia se hubiese sentido desilusionada & 1.6 & .91 & 1 & 1 \\
\hline $\begin{array}{l}\text { 17.Si decidiera abandonar la titulación que estoy cursando mi familia se } \\
\text { sentiría defraudada }\end{array}$ & 2.8 & 1.33 & 3 & 2 \\
\hline $\begin{array}{l}\text { 18.Si decidiera abandonar la titulación que estoy cursando mi familia me } \\
\text { apoyaría }\end{array}$ & 3.4 & 1.14 & 4 & 1 \\
\hline 19.Tengo miedo de decepcionar a mi familia & 3.2 & 1.36 & 3 & 2 \\
\hline $\begin{array}{l}\text { 20.Plantear a mi familia un cambio o abandono de titulación me genera } \\
\text { ansiedad }\end{array}$ & 2.5 & 1.34 & 2 & 3 \\
\hline
\end{tabular}

Continuando con el análisis de los datos y atendiendo al contraste de medias (ver Tabla 2), destaca que las dos variables que hacen referencia a la nota media (superior a 8 en bachillerato y a 9.5 en la EBAU) son las que presentan un mayor número de diferencias significativas, siguiendo ambas la misma tendencia. Así, el alumnado con mejores calificaciones es el que se siente más satisfecho con la elección de titulación, asiste más a clase, está más adaptado (a nivel académico y social), se siente más animado por su familia y percibe que ésta está orgullosa en mayor medida, le apoya y confía en sus decisiones, a la vez que tiene menos miedo a decepcionarla y le genera menos ansiedad plantearle un posible cambio o abandono de titulación.

En cuanto al sexo (ver Tabla 2), los varones piensan más en abandonar los estudios, mientras que las mujeres están más satisfechas con la elección de carrera, tienen una asistencia más elevada, consideran que sus familias se sienten más orgullosas, las apoyan más y confían en sus decisiones. De igual modo, ellas vinculan más su éxito al de sus familias, creen que estas tienen expectativas muy elevadas sobre su futuro académico y manifiestan mayor miedo a decepcionarlas.

Contar con algún tipo de beca, variable directamente relacionada con la dependencia económica de las familias, también muestra diferencias estadísticamente significativas (ver Tabla 2). Así, se reflejan puntuaciones medias superiores en el caso del alumnado que sí percibe la ayuda, en cuestiones como: considerar un gran esfuerzo para la familia costear los estudios, estar más satisfecho con la elección de la carrera, asistir regularmente a clase, percibir que la familia se siente orgullosa y verse más apoyado por esta, ya sea respecto a las decisiones que se toman o ante la posibilidad de abandonar la titulación cursada.

Haber cursado el bachillerato de ciencias también presenta un elevado número de diferencias significativas (ver Tabla 2). El alumnado de ciencias indica, en mayor medida, que la elección de la carrera ha sido exclusivamente suya y que se siente satisfecho con la misma. Por el contrario, los que cursaron otra modalidad, sí muestran una mayor tendencia a que la familia ejerciera presión en dicha elección y que tal decisión fuese por tradición familiar. Igualmente, piensan más en abandonar los estudios, perciben mayores expectativas por parte de la familia, tienen miedo a decepcionarla y creen que, en caso de haber elegido otra carrera, ésta se hubiera sentido desilusionada. 
Por otra parte, tener padres con estudios universitarios presenta pocas diferencias significativas (ver Tabla 2). En el caso de estudiantes con padres universitarios y de sexo masculino, han sufrido mayor presión a la hora de escoger la carrera; mientras que, en el caso de las madres, ha tenido más influencia la elección por tradición familiar. En ambos casos, con padres y madres universitarios, el alumnado tiene mayor miedo a decepcionar a su familia. En cambio, en los alumnos con padres y madres sin estudios superiores, la elección de la carrera ha sido exclusivamente suya y costear los estudios supone un gran esfuerzo para la familia.

\section{Tabla 2}

Diferencias significativas en función de variables sociodemográficas

\begin{tabular}{lccccccc}
\hline \multicolumn{1}{c}{ Ítems } & Sexo & Beca & Ciencias & Media B. & EBAU & Madre & Padre \\
\cline { 2 - 7 } & $\mathrm{p}(\mathrm{d})$ & $\mathrm{p}(\mathrm{d})$ & $\mathrm{p}(\mathrm{d})$ & $\mathrm{p}(\mathrm{d})$ & $\mathrm{p}(\mathrm{d})$ & $\mathrm{p}(\mathrm{d})$ & $\mathrm{p}(\mathrm{d})$ \\
\hline 1.PresiónElección & & & $.002(.30)$ & & & & $.001(.34)$ \\
2.ElecciónFamiliar & & & $.001(.34)$ & & & $.008(.23)$ & $.001(.34)$ \\
3.ElecciónLibre & & & $.026(.22)$ & & & $.034(.18)$ & $.001(.34)$ \\
4.GastoFamilia & & $.000(.37)$ & & $.004(.23)$ & $.002(.24)$ & $.000(.46)$ & $.000(.40)$ \\
5.SatisElección & $.016(.20)$ & $.008(.21)$ & $.004(.22)$ & $.006(.22)$ & $.018(.19)$ & & \\
6.AsistenciaAlta & $.000(.30)$ & $.000(.28)$ & & $.002(.25)$ & $.024(.18)$ & & \\
7.AnsiedadAsistir & & & & $.049(.16)$ & $.003(.24)$ & \\
8.AdaptaAcademi & & & & & $.049(.16)$ & \\
9.AdaptaSocial & & & $.018(.23)$ & & & \\
10.PensarAbandonar & $.028(.18)$ & & & $.014(.20)$ & $.014(.20)$ & & \\
11.FamiliaAnima & & & $.012(.20)$ & $.003(.24)$ & & \\
12.FamiliaOrgullo & $.003(.24)$ & $.014(.20)$ & & $.000(.30)$ & $.001(.28)$ & & \\
13.FamiliaConfía & $.007(.22)$ & $.004(.23)$ & & & & \\
14.FamiliaÉxito & $.027(.18)$ & & $.010(.25)$ & & & & \\
15.FamiliaExpecta & $.044(.16)$ & & $.001(.32)$ & & & \\
16.FamiliaDesilusión & & & & $.007(.21)$ & $.005(.23)$ & & \\
17.FamiliaDefrauda & & & & $.016(.19)$ & & \\
18.FamiliaApoyo & & $.000(.28)$ & $.017(.18)$ & $.011(.20)$ & $.000(.28)$ & $.044(.15)$ & \\
19.FamiliaMiedo & $.005(.24)$ & & & $.019(.19)$ & $.001(.26)$ & & \\
20.FamiliaAnsiedad & & & & & &
\end{tabular}

Profundizando en la relación entre variables, la Tabla 3 permite identificar un elevado número de correlaciones significativas entre aquellas que componen este estudio. Así, destacan por sus valores entre .40 y .75: la influencia entre el ítem 1 (presión familiar en la elección de carrera) y los ítems 2, 3 y 16 (directamente relacionados con la elección de carrera y la familia); la relación positiva entre el conjunto de ítems 11, 12 y 13 (familias que animan, se sienten orgullosas y apoyan en las decisiones); o entre la ansiedad a la hora de plantear un cambio/abandono de titulación (ítem 20) y el miedo a defraudar o decepcionar a la familia (ítems 17 y 19). Especial atención merece la variable referente a la intención de abandonar los estudios (ítem 10), la cual presenta una correlación inversa con la satisfacción con la elección de titulación (ítem 5) y directa con la ansiedad producida ante la idea de acudir a la facultad (ítem 7). Aunque con un coeficiente de correlación menor, pero igualmente significativo, en el análisis de esta variable también tienen relevancia otras como: el nivel de adaptación académico (ítem 8) y social (ítem 9); el ánimo y optimismo mostrado por la familia (ítem 11 ); y que ésta apoye y confíe en las decisiones del alumno (ítem 13). Todas ellas con una relación inversa ante el planteamiento de abandono. 
Tabla 3

Matriz de correlaciones bivariadas

\begin{tabular}{|c|c|c|c|c|c|c|c|c|c|c|c|c|c|c|c|c|c|c|c|}
\hline & 1 & 2 & 3 & 4 & 5 & 6 & 7 & 8 & 9 & 10 & 11 & 12 & 13 & 14 & 15 & 16 & 17 & 18 & 19 \\
\hline 2 & $\begin{array}{l}.41^{\star *} \\
-.51^{\star *}\end{array}$ & $\begin{array}{c}1 \\
-33^{* *}\end{array}$ & 1 & & & & & & & & & & & & & & & & \\
\hline 3 & $\begin{array}{c}-.51^{* *} \\
-0.07\end{array}$ & $\begin{array}{l}-.33^{* *} \\
-14^{* *}\end{array}$ & $\begin{array}{c}1 \\
1\end{array}$ & & & & & & & & & & & & & & & & \\
\hline 4 & $\begin{array}{l}-.07 \\
-2 x^{* \star}\end{array}$ & $-.14^{\star \star \star}$ & $.10^{* *}$ & 1 & & & & & & & & & & & & & & & \\
\hline 5 & $-.22^{* *}$ & $-.11^{* *}$ & $.31^{* *}$ & $.11^{* *}$ & 1 & & & & & & & & & & & & & & \\
\hline 6 & $-.10^{* *}$ & $-.10^{* *}$ & $.08^{*}$ & .03 & $20^{* *}$ & 1 & & & & & & & & & & & & & \\
\hline 7 & $.17^{* *}$ & $.10^{*}$ & $-.13^{* *}$ & .06 & $-.27^{* *}$ & $-.12^{*}$ & 1 & & & & & & & & & & & & \\
\hline 8 & -.05 & -.01 & .03 & $-.09^{*}$ & $.22^{* *}$ & $.15^{\star \star}$ & $-.24^{\star \star}$ & 1 & & & & & & & & & & & \\
\hline 9 & -.07 & -.02 & .04 & -.05 & $.19^{* *}$ & $.09^{*}$ & $-.23^{* *}$ & $.62^{* *}$ & 1 & & & & & & & & & & \\
\hline 10 & $.15^{* *}$ & .06 & $-.18^{* *}$ & -.01 & $-.47^{* *}$ & $-.18^{* *}$ & $.46^{* *}$ & $-.23^{* *}$ & $-.25^{* *}$ & 1 & & & & & & & & & \\
\hline 11 & $-.12^{* *}$ & .00 & .07 & $-.08^{\star}$ & $.18^{* *}$ & $.17^{\star *}$ & $-.21^{\star \star}$ & $.23^{* *}$ & $.25^{* *}$ & $-.24^{\star \star}$ & 1 & & & & & & & & \\
\hline 12 & $-.13^{\star \star}$ & -.02 & $.14^{* *}$ & -.07 & $.20^{* \star}$ & $.16^{* \star}$ & $-.10^{* \star}$ & $.24^{* *}$ & $.24^{* \star}$ & $-.17^{\star \star}$ & $.73^{* *}$ & 1 & & & & & & & \\
\hline 13 & $-20^{* *}$ & -.07 & $.21^{1 *}$ & $-.09^{\star}$ & $.22^{* *}$ & $.23^{* *}$ & $-.15^{\star \star}$ & $.24^{* *}$ & $.27^{* *}$ & $-.22^{\star *}$ & $.71^{* *}$ & $.75^{* \star}$ & 1 & & & & & & \\
\hline 14 & .04 & .02 & .01 & .01 & -.02 & .02 & .07 & .05 & .03 & .03 & $.12^{* *}$ & $.15^{* *}$ & $.09^{* *}$ & 1 & & & & & \\
\hline 15 & $.12^{\star *}$ & $.08^{*}$ & -.06 & .03 & .04 & $.09^{*}$ & .05 & $.11^{* *}$ & .07 & -.01 & $.22^{* *}$ & $.27^{* *}$ & $.24^{* *}$ & $.33^{* *}$ & 1 & & & & \\
\hline 16 & $.47^{* *}$ & $24^{* \star}$ & $-.31^{* *}$ & .03 & $-.12^{* *}$ & $-.13^{* *}$ & $.23^{* *}$ & $-.12^{* *}$ & $-.18^{\star *}$ & $.20^{* *}$ & $-.25^{\star *}$ & $-.22^{\star \star}$ & $-.34^{\star \star}$ & .05 & $.08^{*}$ & 1 & & & \\
\hline 17 & $.21^{* *}$ & $.17^{\star *}$ & $-.18^{* *}$ & .06 & -.07 & $-.13^{* *}$ & $.17^{* *}$ & $-.14^{* \star}$ & $-11^{* *}$ & $.11^{*}$ & $-.24^{\star \star}$ & $-.24^{\star \star}$ & $-.31^{\star \star}$ & $.08^{*}$ & $-.10^{*}$ & $.35^{* *}$ & 1 & & \\
\hline 18 & $-.22^{* *}$ & $-.14^{* *}$ & $.18^{* *}$ & -.05 & .07 & $.10^{*}$ & $-.13^{* *}$ & $.11^{* *}$ & $.10^{*}$ & -.04 & $.27^{* *}$ & $.27^{* *}$ & $.33^{* *}$ & -.01 & -.05 & $-.33^{* *}$ & $-.62^{* *}$ & 1 & \\
\hline 19 & $.18^{* *}$ & .06 & $-.09^{*}$ & $.12^{* *}$ & $-.09^{*}$ & -.04 & $.23^{* *}$ & $-.14^{* *}$ & $-.13^{* *}$ & $.19^{* *}$ & -.03 & -0.1 & $-.10^{*}$ & $.19^{* *}$ & $.22^{* *}$ & $.18^{* *}$ & $.32^{* *}$ & $-.16^{* *}$ & 1 \\
\hline 20 & $.19^{* \star}$ & $.09^{*}$ & $-.15^{* *}$ & $.17^{\star *}$ & $-.18^{\star \star}$ & $-.09^{*}$ & $.27^{* \star}$ & $-.20^{* *}$ & $-.21^{* *}$ & $.28^{* *}$ & $-.20^{* *}$ & $-.17^{* *}$ & $-.26^{* *}$ & $.10^{* *}$ & .06 & $.22^{* *}$ & $.41^{* *}$ & $-.30^{* *}$ & $.57^{* *}$ \\
\hline
\end{tabular}

Nota: ${ }^{*} p<.05 ;{ }^{* *} p<.01$

Por último, con el objetivo de indagar aún más en alguna de las variables que han resultado relevantes (a nivel teórico y estadístico), se plantean dos modelos predictivos; uno centrado en la idea de abandonar los estudios (Pienso, habitualmente, en abandonar mis estudios y la titulación actual) y otro en la influencia familiar ante la ansiedad que puede producir informar de la intención de abandonar (Plantear a mi familia un cambio o abandono de titulación me genera ansiedad).

La primera de ellas, queda explicada por un modelo predictivo (ver Tabla 4) integrado por tres variables, que permite explicar el $60 \%$ de la varianza; entre las que destaca sentirse satisfecho con la elección de titulación. También parecen influir la ansiedad que produce pensar en ir a la facultad y plantear a la familia un cambio/abandono de titulación.

Tabla 4

Modelo predictivo de la variable: Pienso, habitualmente, en abandonar mis estudios y la titulación actual

\begin{tabular}{cccc}
\hline Modelo & $\mathrm{R}$ & Beta & Sig. \\
\hline 1 & .47 & -.36 & .000 \\
2 & .58 & .33 & .000 \\
3 & .60 & .13 & .000 \\
\hline
\end{tabular}

Predictores: 1-Me siento satisfecho con la elección de mi titulación; 2-Me produce ansiedad pensar en ir a la facultad; 3-Plantear a mi familia un cambio o abandono de titulación me genera ansiedad.

Por otro lado, tal ansiedad ante la idea de plantear a la familia el cambio o abandono de titulación (ver Tabla 5), queda explicada por un modelo predictivo compuesto por otras tres variables, que permite explicar el $64 \%$ de la varianza. Entre ellas la que más aporta es el miedo a decepcionar a la familia, seguida de la convicción de que ésta se sentiría defraudada si se abandonan los estudios y, con menos peso, plantearse habitualmente tal abandono.

\section{Tabla 5}

Modelo predictivo de la variable: Plantear a mi familia un cambio o abandono de titulación me genera ansiedad

\begin{tabular}{cccc}
\hline Modelo & $\mathrm{R}$ & Beta & Sig. \\
\hline 1 & .57 & .46 & .000 \\
2 & .62 & .24 & .000 \\
3 & .64 & .17 & .000 \\
\hline
\end{tabular}

Predictores: 1-Tengo miedo de decepcionar a mi familia; 2-Si decidiera abandonar la titulación que estoy cursando mi familia se sentiría defraudada; 3-Pienso, habitualmente, en abandonar mis estudios y la titulación actual. 


\section{Discusión y Conclusiones}

En la actualidad uno de los fenómenos educativos que más trascendencia investigadora está teniendo es el abandono de los estudios en las distintas etapas académicas (obligatoria y postobligatoria). Por ello, en los últimos años han aumentado notablemente los estudios sobre las múltiples variables que inciden en este hecho. La mayoría de ellos, enmarcados en la etapa de secundaria (Fan \& Walters, 2014; Rumberger \& Rotermund, 2012), han dado cuenta del papel amortiguador que tienen diversas variables personales del estudiante (rendimiento, etc.) para hacer que éste progrese académicamente y lleve a término sus estudios obligatorios. Sin embargo, pocas son las investigaciones (Fan \& Williams, 2010; Ross, 2016) que analizan en esta etapa el carácter multicausal del abandono teniendo en cuenta la familia, y menos aún en las etapas educativas postobligatorias (bachillerato y universidad). Al objeto de arrojar un poco más de luz sobre este fenómeno dentro del contexto de la educación superior se ha planteado el siguiente estudio. En el mismo, y ateniendo al carácter poliédrico del abandono, se han medido diversas variables tanto de la esfera personal del estudiante (a saber: rendimiento académico previo a la entrada al Grado, motivos de elección de la carrera y nivel de adaptación al entorno universitario) como de su contexto más próximo: el familiar (datos sociodemográficos, percepción de apoyo de la familia y expectativas familiares); y cómo la relación entre ellas puede influir en su intención de dejar la carrera.

Comenzando con el grupo de variables personales analizadas, los resultados aquí hallados demuestran que la nota de acceso a la titulación no solo correlaciona positivamente con otros indicadores de permanencia ampliamente validados en la literatura científica como la satisfacción con la carrera elegida (Cabrera, Bethencourt, González, \& Álvarez, 2006), la asistencia a clases y la adaptación académica (Esteban et al., 2017); sino también con otras variables familiares. En efecto, los datos obtenidos reflejan que, aquellos alumnos que han ingresado a la universidad con buenas calificaciones en el bachillerato y en la prueba de acceso, son los que más perciben que sus familias les apoyan y confían en sus decisiones, que se sienten orgullosas del camino académico emprendido y que, en consecuencia, les animan a seguir adelante con sus planes futuros. Igualmente, son aquellos que sienten menor temor a plantearles un posible abandono de titulación y a decepcionarlas si no cumplen las expectativas que de ellos tenían.

En cuanto a la elección del grado académico, los alumnos afirman, en su mayoría, no haber estado condicionados por algún tipo de presión o tradición familiar, pudiendo tomar una decisión más autónoma y acorde a sus intereses académico-profesionales. Estos resultados coinciden con los obtenidos por Rodríguez, Peña e Inda (2015) quienes, en una muestra de estudiantes de secundaria españoles, concluyen que casi la totalidad de ellos fundamentan su decisión basándose principalmente en el gusto y preferencia personal. Además, encuentran que, si bien los padres de los estudiantes constituyen para ellos una fuente de apoyo importante durante este proceso, a la postre son ellos mismos quienes toman las riendas de su elección final. En concordancia con los resultados obtenidos por Bethencourt et al. (2008), se concluyó que de las variables, aquí estudiadas, la que mejor predice si un alumno tiene o no intenciones de abandonar sus estudios es la satisfacción con la titulación. Cabe destacar que esta variable, si bien no figura dentro de aquellas directamente vinculadas al contexto familiar del estudiante, nuestros resultados revelan que guarda relación con el papel ejercido por la familia durante la etapa previa al ingreso a la universidad y, específicamente, durante su proceso de elección de carrera. Así, nuestros hallazgos indican que aquellos estudiantes que manifiestan haber elegido la titulación que están cursando por voluntad propia, son los que más satisfechos se sienten con la carrera matriculada, mejor se adaptan (académica y socialmente) y menor intención tienen de abandonarla. Estos resultados van en línea con los obtenidos por Girelli et al. (2018), quienes demuestran que aquellos alumnos que perciben que sus padres promueven su autonomía individual, eligen estudiar en la universidad por motivos propios, lo que se relaciona con una menor intención de abandonar la carrera durante el primer año y, en consecuencia, con una mejor adaptación académica.

En relación al sexo, algunas investigaciones han demostrado que las mujeres no sólo tienen mayores niveles de auto-disciplina y auto-control (Duckworth \& Seligman, 2005), sino también una mayor capacidad para hacer frente a las dificultades que se les puedan presentar durante su transcurso académico, lo que les permite obtener un mejor rendimiento y prosperar satisfactoriamente en sus estudios superiores (Vera, 2018). Esto podría explicar por qué en nuestra investigación fueron también las jóvenes quienes manifestaron una menor intención de dejar su carrera sin culminar. Además, ello no resulta extraño tomando en consideración que es este mismo colectivo el que más satisfacción presenta con la carrera elegida, tiene una asistencia más elevada a las clases y un mayor apoyo familiar percibido. No obstante, estos resultados contrastan con los de González-Ramírez y Pedraza-Navarro (2017) quienes, en una muestra de estudiantes universitarios de primer curso, encontraron que eran las chicas quienes más tendían a desertar debido a la aparición de situaciones 
socio-familiares (como por ejemplo el nacimiento de un hijo) que, al interferir con la normal continuidad de sus estudios, les llevaban a tomar la decisión final de abandonarlos.

Continuando ahora con el grupo de variables vinculadas al contexto familiar, en concordancia con los resultados obtenidos por Fernández-García et al. (2016), en el presente estudio los alumnos consideran, en gran medida, que sus familiares tienen unas expectativas muy elevadas sobre su futuro académico. A su vez, bastantes de ellos, tienen la creencia (falsa o no) de que éstas podrían sentirse defraudadas si en algún momento decidieran abandonar la carrera. Ante este escenario, no es extraño que varios hayan reportado sentir miedo ante la posibilidad de decepcionar a su familia, y en el caso de aquellos que piensan habitualmente en dejar la titulación, tampoco es sorprendente que les genere ansiedad plantearles tal decisión. Y esta preocupación que genera al alumno comunicar esta decisión a la familia lo hace más propenso a considerar como una alternativa el dejar el Grado. Por lo tanto, la ansiedad que produce esta situación en el estudiante parece ser un factor detonante y acelerador de este proceso. Asimismo, los datos ponen de manifiesto que este temor del alumnado a decepcionar a sus familias se evidencia, principalmente, en aquellos estudiantes cuyos padres poseen estudios universitarios. Esto podría estar relacionado con la preocupación de los jóvenes por seguir los estándares académicos de los progenitores, sobre todo en los casos en los que se proviene de familias de niveles socioeconómicos altos, donde parece que el mensaje que se transmite a los hijos es el de la necesidad de que sean exitosos en la vida, siendo la preparación académico-profesional la vía para conseguirlo (Asare, Nicholson, \& Stein, 2017). En cambio, en las familias donde los hijos son la primera generación que accede a la universidad, el deseo de éstas está más ligado a la expectativa de que sus hijos puedan tener las oportunidades que ellos no tuvieron; $y$ en el caso de aquellas que pertenecen a estratos socioeconómicos menos favorecidos a la esperanza de que éstos puedan ayudarles a mejorar la situación económica en la que se encuentra la familia (Gonzáles, 2016).

Las diferencias por sexo patentes en esta investigación evidenciaron, nuevamente, discrepancias en lo que respecta a las expectativas de éxito que depositan las familias sobre el alumnado. Los resultados obtenidos indican que las chicas perciben de sus familiares unas expectativas más elevadas sobre su futuro académico, resultado que difiere de lo obtenido por Root et al. (2003), quienes encontraron que las expectativas de los padres eran más altas para los hijos varones. En definitiva, tanto para las chicas como para los chicos, estos hallazgos merecen especial atención dado que, tal y como señalan Cabrera, Bethencourt, Álvarez y González (2006) estas altas aspiraciones de las familias pueden suponer una fuente importante de presiones y tensiones para el alumno que podrían poner en riesgo su permanencia en los estudios.

El hecho de haber sido estudiantes exitosos (obtener buenas calificaciones) en las etapas educativas previas se relaciona con una mejor adaptación a la universidad y, en concordancia con lo encontrado por Fajardo, Maestre y Polo (2017), con la percepción de una implicación positiva de la familia en su progreso académico, lo cual podría funcionar como un factor protector contra la deserción. De hecho, los resultados aquí obtenidos lo corroboran, ya que los alumnos que mejores notas de acceso a la titulación han tenido, perciben este tipo implicación familiar (ánimo, optimismo, apoyo y confianza), y, además, son los que mejor se adaptan al contexto universitario y presentan menor intención de abandonar sus estudios, como así también lo demuestran Ojeda, Navarro y Morales (2011).

En síntesis, la presente investigación ha puesto de relieve que, además de las variables personales del estudiante ampliamente estudiadas, variables del ámbito familiar tales como el apoyo y las expectativas, juegan un papel importante en el planteamiento de abandono de los estudios en los jóvenes que se encuentran en la etapa educativa universitaria. Por tanto, el principal aporte de este estudio es que ha ido más allá de la investigación de las variables familiares socio-demográficas hasta ahora analizadas, ampliando su objeto de investigación a otras variables del contexto familiar, las cuales pueden ser cruciales tanto para la adaptación al entorno universitario como para la persistencia en el mismo. Sin embargo, dadas las características de este estudio sería deseable seguir investigando en esta línea con una muestra más amplia y heterogénea, para continuar indagando en la influencia que el contexto familiar puede presentar en la población en riesgo de abandono de cara a ofrecer acciones y medidas eficaces, desde el propio seno familiar, que favorezcan la permanencia de los jóvenes en sus estudios superiores. Además, sería interesante para futuros trabajos perfeccionar los instrumentos de recogida de información hasta ahora utilizados e incorporar información cualitativa y la visión de otras fuentes como el profesorado y la propia familia, lo que posibilitaría triangular la información obteniendo una visión más próxima a la realidad. 


\section{Referencias}

Álvarez-Pérez, P. R., \& López-Aguilar, D. (2017). Estudios sobre deserción académica y medidas orientadoras de prevención en la Universidad de la Laguna (España). Revista Paradigma, 38(1), 48-71.

Asare, S., Nicholson, H., \& Stein, S. (2017). You can't ignore us: what role does family play in student engagement and alienation in a Ghanaian university? Journal of Higher Education Policy and Management, 39(6), 593-606. https://doi.org/10.1080/1360080X.2017.1377968

Bernardo, A., Cerezo, R., Núñez, J. C., Tuero, E., \& Esteban, M. (2015). Predicción del abandono universitario: Variables explicativas y medidas de prevención. Revista Fuentes, 16, 63-84.

https://doi.org/10.12795/revistafuentes.2015.116.03

Bethencourt, J. T., Cabrera, L., Hernández, J. A., Álvarez, P., \& González, M. (2008). Variables psicológicas y educativas en el abandono universitario. Revista Electrónica de Investigación Psicoeducativa, 6(3), 603-622. Cabrera, L., Bethencourt, J. T., Álvarez, P., \& González, M. (2006). El problema del abandono de los estudios universitarios. Revista Electrónica de Investigación y Evaluación Educativa, 12(2), 171-203.

Cabrera, L., Bethencourt, J. T., González, M., \& Álvarez, P. (2006). Un estudio transversal retrospectivo sobre prolongación y abandono de estudios universitarios. Revista Electrónica de Investigación y Evaluación Educativa, 12(1), 105-127.

Comisión Europea (2012) Europa 2020: La estrategia Europea de Crecimiento. Recuperado de http://bit.ly/2/KMgxh Cronbach, L. J., \& Shavelson, R. J. (2004). My current thoughts on coefficient alpha and successor procedures. Educational and Psychological Measurement, 64(3), 391-418. https://doi.org/10.1177/0013164404266386 De Ortuzar, V., \& Di Meglio, M. (2016). Aportes de la re-orientación como estrategia de retención universitaria. Orientación y Sociedad, 16, 153-163.

Duckworth, A. L., \& Seligman M. E. P. (2005). Self-Discipline Outdoes IQ in Predicting Academic Performance of Adolescents. Psychological Science, 16(12), 939-944. https://doi.org/10.1111/j.1467-9280.2005.01641.x Esteban, M., Bernardo, A., Tuero, E., Cervero, A., \& Casanova, J. (2017). Variables that influence academic performance and university persistence. European Journal of Education and Psychology, 10(2), 75-81. http://doi.org/10.1016/j.ejeps.2017.07.003

Esteban, M., Bernardo, A., Tuero, E., Cervero, A., \& Núñez, J. C. (2016). El contexto sí importa: Identificación de relaciones entre el abandono de titulación y variables contextuales. European Journal of Education and Psychology, 9(2), 47-88. http://doi.org/10.1016/j.ejeps.2015.06.001

Fan, W., \& Williams, C. M. (2010). The effects of parental involvement on student's academic self-efficacy, engagement and intrinsic motivation. Educational Psychology, 30(1), 53-74. https://doi.org/10.1080/01443410903353302 Fan, W., \& Walters, C. A. (2014). School motivation and high school dropout: The mediating role of educational expectation. British Journal of Educational Psychology, 84, 22-39. https://doi.org/10.1111/bjep.1200

Fajardo, F., Maestre, M., Felipe, E., León del Barco, B., \& Polo del Río, M. I. (2017). Análisis del rendimiento académico de los alumnos de Educación Secundaria Obligatoria según las variables familiares. Educación XX1, 20(1), $209-232$. https://doi.org/10.5944/educXX1.14475

Fernández-García, C. M., García-Pérez, O., \& Rodríguez-Pérez, S. (2016). Los padres y madres ante la toma de decisiones académicas de los adolescentes en la Educación Secundaria: un estudio cualitativo. Revista Mexicana de Investigación Educativa, 21(71), 1111-1133.

García, A. M., \& Adrogué, C. (2015). Abandono de los estudios universitarios: dimensión, factores asociados y desafíos para la política pública. Revista Fuentes, 16, 85-106. https://doi.org/10.12795/revistafuentes.2015.116.04

Ghignoni, E. (2017). Family background and university dropouts during the crisis: The case of Italy. Higher Education, 73, 127-151. https://doi.org/10.1007/s10734-016-0004-1

Girelli, L., Alivernini, F., Lucidi, F., Cozzolino, M., Savarese, G., Sibilio, M., \& Salvatore, S. (2018). Autonomy Supportive Contexts, Autonomous Motivation, and Self-Efficacy Predict Academic Adjustment of First-Year University Students. Frontiers in Education, 3(95), 1-11. https://doi.org/10.3389/feduc.2018.00095

Gonzáles, A. M. (2016). Parental Influence and Relationship among First Generation College Students Attending Community College (Tesis Doctoral). Texas Tech University, Texas, USA.

González, M. C., Álvarez, P. R. Cabrera, L., \& Bethencourt, J. T. (2007). El abandono de los estudios universitarios: factores determinantes y medidas preventivas. Revista Española de Pedagogía, 65(236), 71-85.

González, L. E., \& Uribe, D. (2018). Estimaciones sobre la "repitencia" y deserción en la educación superior chilena. Consideraciones sobre sus implicaciones. Calidad en la Educación, 17, 75-90. https://doi.org/10.31619/caledu.n17.408 González-Ramírez, T., \& Pedraza-Navarro, I. (2017). Variables sociofamiliares asociadas al abandono de los estudios universitarios. Educatio Siglo XXI, 35(2), 365-388. https://doi.org/10.6018/j/298651

Iñíguez, T., Elboj, C., \& Valero, D. (2016). La Universidad del Espacio Europeo de Educación Superior ante el abandono de los estudios de grado. Causas y Propuestas Estratégicas de Prevención. Educar, 52(2), 285-313.

https://doi.org/10.5565/rev/educar.674

Motl, T., Multon, K., \& Zhao, F. (2018). Persistence at a Tribal University: Factors Associated With Second Year Enrrollment. Journal of Diversity in Higher Education, 11(1), 51-66. https://doi.org/10.1037/dhe0000034

Nervi, C., Rodríguez, J., \& Osada, J. (2015). Deserción universitaria durante el primer año de estudios. Fundación Educación Médica, 18(2), 93. 
Ojeda, L., Navarro, R., \& Morales, A. (2011). The role of La Familia on Mexican American Men's College Persistence Intentions. Psychology of Men \& Masculinity, 12(3), 216-229. https://doi.org/10.1037/a0020091

Rodrigo, M. F., Molina, J. G., García-Ros, R., \& Pérez-González-F. (2012). Efectos de interacción en la predicción del abandono en los estudios de Psicología. Anales de Psicología, 28(1), 113-119.

Rodríguez, M. C., Peña, J. V., \& Inda, M. M. (2016). Esto es lo que me gusta y lo que voy a estudiar: Un estudio cualitativo sobre la toma de decisiones académicas en bachillerato. Revista Complutense de Educación, 27(3), 13511368. https://doi.org/10.5209/rev_RCED.2016.v27.n3.48518

Root, S., Rudawski, A., Taylor, M., \& Rochon, R. (2003). Attrition of Hmong Students in Teacher Education Programs. Bilingual Re-search Journal, 27(1), 137-48. https://doi.org/10.1080/15235882.2003.10162595

Ross, T. (2016). The differential effects of parental involvement on high school completion and postsecondary attendance. Education Policy Analysis Archives, 24(30), 1-38. https://doi.org/10.14507/epaa.v24.2030

Rumberger, R. W., \& Rotermund, S. (2012). The relationship between engagement and high school dropout. In S. L. Christenson, A. L. Reschly \& C. Wylie (Eds.), Handbook of Research on Student Engagement (pp. 491-513). New York: Springer.

Silva, M. (2011). El primer año universitario. Un tramo crítico para el éxito académico. Perfiles Educativos, 33, $102-114$. Strom, R. E., \& Savage, M. W. (2014). Assessing the Relationships Between Perceived Support from Close Others, Goal Commitment, and Persistence Decisions at the College Level. Journal of College Student Development, 55(6), 531-547. https://doi.org/10.1353/csd.2014.0064

Stubbs, N. S., \& Maynard, D. M. B. (2017). Academic self-efficacy, school engagement and family functioning, among postsecondary students in the Caribbean. Journal of Child and Family Studies, 26(3), 792-799. httpss://doi.org/10.1007/s10826-016-0595-2

Tinto, V. (1975). Dropout from Higher Education: A Theoretical Synthesis of Recent Research. Journal of Higher Education, 45(1), 89-125. https://doi.org/10.3102/00346543045001089

Vera, S. (2018). Resiliencia, Género y Rendimiento Académico en los Estudios Universitarios (Tesis Doctoral). Universidad de Sevilla, Sevilla, España. 